JOURNAL OF

SYNCHROTRON

RADIATION

ISSN 1600-5775

Received 5 February 2018

Accepted 5 July 2018

Edited by S. Svensson, Uppsala University, Sweden

Keywords: harmonic rejection; monochromator; coherent X-ray scattering; SAXS; XAFS.

Supporting information: this article has supporting information at journals.iucr.org/s

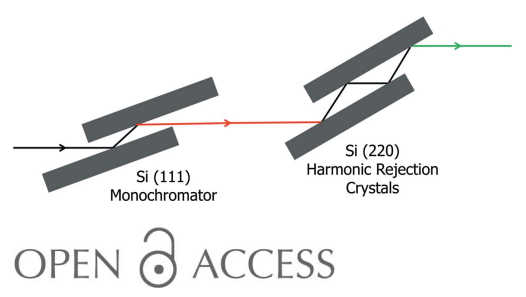

\section{High-efficiency coherence-preserving harmonic rejection with crystal optics}

\author{
Fan Zhang, ${ }^{a *}$ Andrew J. Allen, ${ }^{a}$ Lyle E. Levine, ${ }^{a}$ Gabrielle G. Long, ${ }^{a, b}$ \\ Ivan Kuzmenko ${ }^{b}$ and Jan Ilavsky ${ }^{\text {b* }}$
}

\begin{abstract}
${ }^{a}$ Material Measurement Laboratory, National Institute of Standards and Technology, 100 Bureau Drive, Gaithersburg, MA 20899, USA, and ${ }^{\mathbf{b}}$ X-ray Science Division, Advanced Photon Source, Argonne National Laboratory, Argonne, IL 60439, USA. *Correspondence e-mail: fan.zhang@nist.gov, ilavsky@aps.anl.gov
\end{abstract}

This work reports a harmonic-rejection scheme based on the combination of $\mathrm{Si}(111)$ monochromator and $\mathrm{Si}(220)$ harmonic-rejection crystal optics. This approach is of importance to a wide range of X-ray applications in all three major branches of modern X-ray science (scattering, spectroscopy, imaging) based at major facilities, and especially relevant to the capabilities offered by the new diffraction-limited storage rings. It was demonstrated both theoretically and experimentally that, when used with a synchrotron undulator source over a broad range of X-ray energies of interest, the harmonic-rejection crystals transmit the incident harmonic X-rays on the order of $10^{-6}$. Considering the flux ratio of fundamental and harmonic X-rays in the incident beam, this scheme achieves a total flux ratio of harmonic radiation to fundamental radiation on the order of $10^{-10}$. The spatial coherence of the undulator beam is preserved in the transmitted fundamental radiation while the harmonic radiation is suppressed, making this scheme suitable not only for current third-generation synchrotron sources but also for the new diffraction-limited storage rings where coherence preservation is an even higher priority. Compared with conventional harmonicrejection mirrors, where coherence is poorly preserved and harmonic rejection is less effective, this scheme has the added advantage of lower cost and footprint. This approach has been successfully utilized at the ultra-small-angle X-ray scattering instrument at the Advanced Photon Source for scattering, imaging and coherent X-ray photon correlation spectroscopy experiments. With minor modification, the harmonic rejection can be improved by a further five orders of magnitude, enabling even more performance capabilities.

\section{Introduction}

Synchrotron X-ray sources and the experimental capabilities that they enable are increasingly important in many scientific fields, including physics, materials science, chemistry, environmental science and biology. For most synchrotron-based experimental techniques, monochromatization of the X-ray radiation is required. Crystal-based monochromators are essential components of modern X-ray beamline design which, when properly configured in accordance with source characteristics, can deliver high-brilliance X-rays of specific wavelength (energy) to the sample position. The monochromatization process follows Bragg's law, $n \lambda=2 d \sin \left(\theta_{\mathrm{B}}\right)$, where $n$ is a positive integer, $\lambda$ is the X-ray wavelength, $d$ is the lattice spacing of the monochromator crystals and $\theta_{\mathrm{B}}$ is one half of the diffraction angle $2 \theta_{\mathrm{B}}$. Following Bragg's law, it is obvious that the monochromator allows through not only $\mathrm{X}$-rays of fundamental radiation with wavelength $\lambda$ (where $n=1$ ) but also harmonic radiation with wavelength $\lambda / n$ (excluding crystallographically forbidden reflections) resulting from higher orders of diffraction. For many applications the 
intensity of the harmonic radiation relative to the fundamental radiation can be significant, and this can lead to distortions and errors in the experimental data. Rejection of the harmonic radiation is desirable and in many cases necessary in techniques across modern X-ray science.(Gauthier et al., 1999; Paterson et al., 2011).

A variety of methods have been developed to suppress harmonic radiation, including crystal detuning of a doublecrystal monochromator (Bonse et al., 1976; Hou, 2005), totalreflection mirrors (Latimer et al., 1995; Hastings et al., 1978), asymmetric bent-Laue crystals (Karanfil et al., 2004), compound refractive lenses (Polikarpov et al., 2014a,b) and undulator segmentation (Tanaka \& Kitamura, 2002). Among these, crystal detuning and harmonic-rejection X-ray mirrors are the most common. Crystal detuning relies on the fact that the X-ray refractive index of a given material is energy dependent while the bandwidth of the fundamental radiation can be significantly larger than those of the harmonic radiation. Therefore, by detuning the second monochromator crystal slightly, the flux of harmonic radiation can be suppressed by $10^{-2}$ to $10^{-3}$ of their original values (Hou, 2005). This level of harmonic rejection, though good enough sometimes, is often inadequate, leading to the need for other harmonic-rejection devices, most commonly total-reflection $\mathrm{X}$-ray mirrors in tandem with crystal detuning to further suppress harmonic radiation. These X-ray mirrors are often coated with a high-atomic-number (high- $Z$ ) material, and can be oriented at a selected incident angle so that incident X-rays below a specific energy are totally reflected while harmonic radiation is absorbed. Such mirrors generally have multiple stripes of different reflecting materials to enable harmonic rejection over a wide energy range. Their harmonic-rejection efficiency, defined as the flux ratio of harmonic radiation to fundamental radiation, can reach the order of $10^{-3}$ (Bilderback \& Hubbard, 1982). Combining crystal detuning with total-reflection X-ray mirrors, a harmonic-rejection efficiency ranging from $10^{-5}$ to $10^{-6}$ can be achieved (Lingham et al., 1996).

With the increasing brightness and coherence of new X-ray sources such as diffraction-limited storage rings and X-ray free-electron lasers, X-ray optics that can define, optimize and preserve beam characteristics, particularly beam coherence, are desirable. Many scientific opportunities enabled by these new sources are highly sensitive to the wavefront distortions caused by X-ray optics. For example, it is estimated that, for the purpose of coherence preservation of hard X-rays, X-ray mirrors need to have a surface finish with slope errors less than $50 \mathrm{nrad}$ and root-mean-square height errors less than $0.5 \mathrm{~nm}$ over mirror dimensions approaching $1 \mathrm{~m}$ in length along the beam-propagation direction (Pardini et al., 2015; Mills \& Padmore, 2013). These stringent requirements and high costs associated with constructing mirrors with such low surface figure errors and roughness present major challenges.

In this paper, we offer a highly effective mirrorless harmonic-rejection scheme that has been successfully utilized at the Advanced Photon Source (APS), Argonne National Laboratory, Argonne, IL, USA. This scheme exploits a crystal mismatch between the monochromator crystals and BonseHart-type harmonic-rejection crystals. We demonstrate that this scheme can suppress the intensity of harmonic radiation to $\sim 10^{-10}$ of the intensity of the fundamental radiation over a wide energy range, while preserving the spatial coherence of the X-ray beam.

\section{Theoretical considerations}

The dynamical diffraction of X-rays by a perfect single crystal is well understood. In this paper, we follow the treatment by Als-Nielsen \& McMorrow (2011). The amplitude, $r$, of the single-crystal reflectivity curve, as a function of the real part of $x_{\mathrm{c}}, \operatorname{Re}\left(x_{\mathrm{c}}\right)$, follows

$$
r\left[\operatorname{Re}\left(x_{\mathrm{c}}\right)\right]= \begin{cases}x_{\mathrm{c}}-\left(x_{\mathrm{c}}{ }^{2}-1\right)^{1 / 2} & \text { for } \operatorname{Re}\left(x_{\mathrm{c}}\right) \geq 1, \\ x_{\mathrm{c}}-i\left(1-x_{\mathrm{c}}\right)^{1 / 2} & \text { for }\left|\operatorname{Re}\left(x_{\mathrm{c}}\right)\right| \leq 1, \\ x_{\mathrm{c}}+\left(x_{\mathrm{c}}{ }^{2}-1\right)^{1 / 2} & \text { for } \operatorname{Re}\left(x_{\mathrm{c}}\right) \leq-1,\end{cases}
$$

where $x_{\mathrm{c}}=\pi(\varsigma / g)-\left(g_{0} / g\right), \varsigma=\Delta \lambda / \lambda$ is the relative bandwidth in wavelength $\lambda$,

and

$$
g_{0}=\left(\frac{2 d^{2} r_{0}}{v_{\mathrm{c}}}\right)\left[\sum_{j}\left(Z_{j}+f_{j}^{\prime}+i f_{j}^{\prime \prime}\right)\right]
$$

$$
g=\left(\frac{2 d^{2} r_{0}}{v_{\mathrm{c}}}\right)\left\{\sum_{j}\left[f_{j}^{0}(\mathbf{q})+f_{j}^{\prime}+i f_{j}^{\prime \prime}\right] \exp \left(i \mathbf{q} \cdot \mathbf{r}_{j}\right)\right\} .
$$

Here $v_{\mathrm{c}}$ is the volume of the unit cell, $r_{0}$ is the scattering amplitude per electron, $d$ is the lattice spacing, $f_{j}^{\prime}$ and $f_{j}^{\prime \prime}$ are the real and imaginary parts of the dispersion correction to the atomic scattering length $f_{j}^{0}(\mathbf{q})$. Here, $q=4 \pi / \lambda \sin (\theta)$, where $\theta$ is one half of the scattering angle $2 \theta$. We calculated the energydependent $f_{j}^{\prime}$ using the scattering contrast factor calculator in the small-angle scattering analysis package Irena (Ilavsky \& Jemian, 2009; Cromer \& Liberman, 1981). The crystal Darwin curve (i.e. the intensity reflectivity curve), expressed as a function of $\left(\theta-\theta_{\mathrm{B}}\right)$, is derived from the square of the amplitude given in equation (1). Based on this notation, the angular full width at half-maximum (FWHM) of the Darwin curve follows

$$
\zeta^{\mathrm{FWHM}}=\left[\frac{3}{2 \sqrt{2}}\right] \frac{4 d^{2} r_{0}}{\pi v_{\mathrm{c}}}|F(\mathbf{q})| \tan \left(\theta_{\mathrm{B}}\right),
$$

where $|F(\mathbf{q})|$ is the unit-cell structure factor in the diffraction direction and is energy dependent. An important feature of the Darwin curve is that its center displays a small angular offset from the Bragg angle. Mathematically, this offset is equal to

$$
\theta-\theta_{\mathrm{B}}=\frac{2 d^{2} r_{0}}{\pi v_{\mathrm{c}}}\left|F_{0}\right| \tan \left(\theta_{\mathrm{B}}\right)
$$

where $F_{0}$ is the unit-cell structure factor in the forward direction. For a given Bragg angle, the higher the X-ray energy, the smaller the angular offset and the smaller the FWHM of the Darwin curve. Thus, it is important to note that even nondispersive crystal optics exhibit a small energy dispersion for different crystal reflections. 
Our scheme exploits this offset by regarding the Darwin curve as an angular filter (Zhang et al., 2008). This scheme is illustrated in Fig. 1, where $\mathrm{X}$-rays are diffracted twice off the $\mathrm{Si}(111)$ monochromator crystals and four times by the $\mathrm{Si}(220)$ harmonicrejection crystals. The mismatch between the monochromator crystal diffraction (111) and the harmonicrejection crystal diffraction (220) is critical to this scheme. The $\mathrm{Si}(111)$ crystal passes spectral contamination following third, fourth, fifth... order diffractions $(n=3,4,5 \ldots)$. For an undulator gap optimized for fundamental radiation, the X-ray flux of the harmonic radiation drops precipitously as the $\mathrm{X}$-ray energy increases. Notably, second-order diffraction is forbidden for an $\mathrm{Si}(111)$ monochromator. Thus, we consider only fundamental radiation with the wavelength $\lambda$ and harmonic radiation with $\lambda / 3$, and we neglect further higherorder harmonic radiation as a result of their much lower flux. As we shall explain in detail later, $\mathrm{Si}(111)$ and $\mathrm{Si}(220)$ crystal diffractions possess different relative angular offsets between the diffracted fundamental radiation and diffracted harmonic radiation. This point forms the basis of the highly effective harmonic-rejection scheme described in this paper.

We first inspect the general characteristics of the crystal Darwin curves. An example is shown in Fig. 2(a), which compares the calculated crystal Darwin curves as a function of angular offset from the Bragg angles for fundamental radiation of $21 \mathrm{keV} \mathrm{X}$-rays and harmonic radiation of $63 \mathrm{keV}$ $\mathrm{X}$-rays (third-order diffraction). The angular offset $\theta_{\mathrm{c}}-\theta_{\mathrm{B}}=\left(\chi_{\mathrm{c}} g+g_{0}\right) / \pi \tan \left(\theta_{\mathrm{c}}\right)$. Here, $21 \mathrm{keV} \mathrm{X}$-rays are diffracted by $\mathrm{Si}(111)$ planes and $63 \mathrm{keV}$ X-rays are diffracted by $\operatorname{Si}(333)$ planes with the same Bragg angle $\theta_{\mathrm{B}}$. The parameters used to calculate these Darwin curves are listed in Table 1. It is apparent that the Darwin curve is narrower and its center deviates from $\theta_{\mathrm{B}}$ by a smaller amount for harmonic radiation than it does for fundamental radiation. The same statements can be made for Fig. 2(b), which shows the calculated crystal Darwin curves as a function of the angular offset from the Bragg angle for fundamental radiation of $21 \mathrm{keV}$ $\mathrm{X}$-rays and harmonic radiation of $63 \mathrm{keV} \mathrm{X}$-rays diffracted by the $\mathrm{Si}(220)$ and $\mathrm{Si}(660)$ planes, respectively.

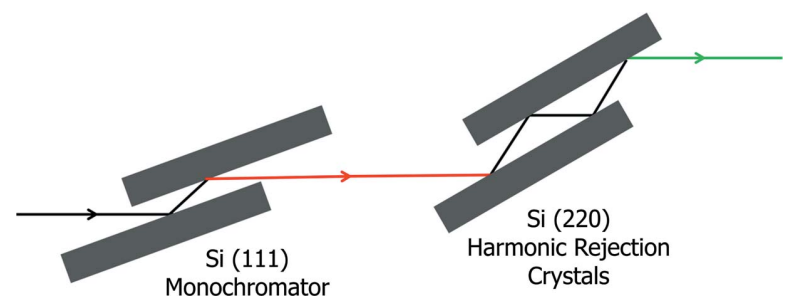

Figure 1

Illustration of the harmonic-rejection scheme reported in this paper. $\mathrm{X}$-rays are reflected twice by the $\mathrm{Si}(111)$ monochromator crystals and four times by the $\mathrm{Si}(220)$ harmonic-rejection crystals. The two crystals in the $\mathrm{Si}(111)$ monochromator and the two $\mathrm{Si}(220)$ crystals of the harmonicrejection pair are perfectly parallel to each other.
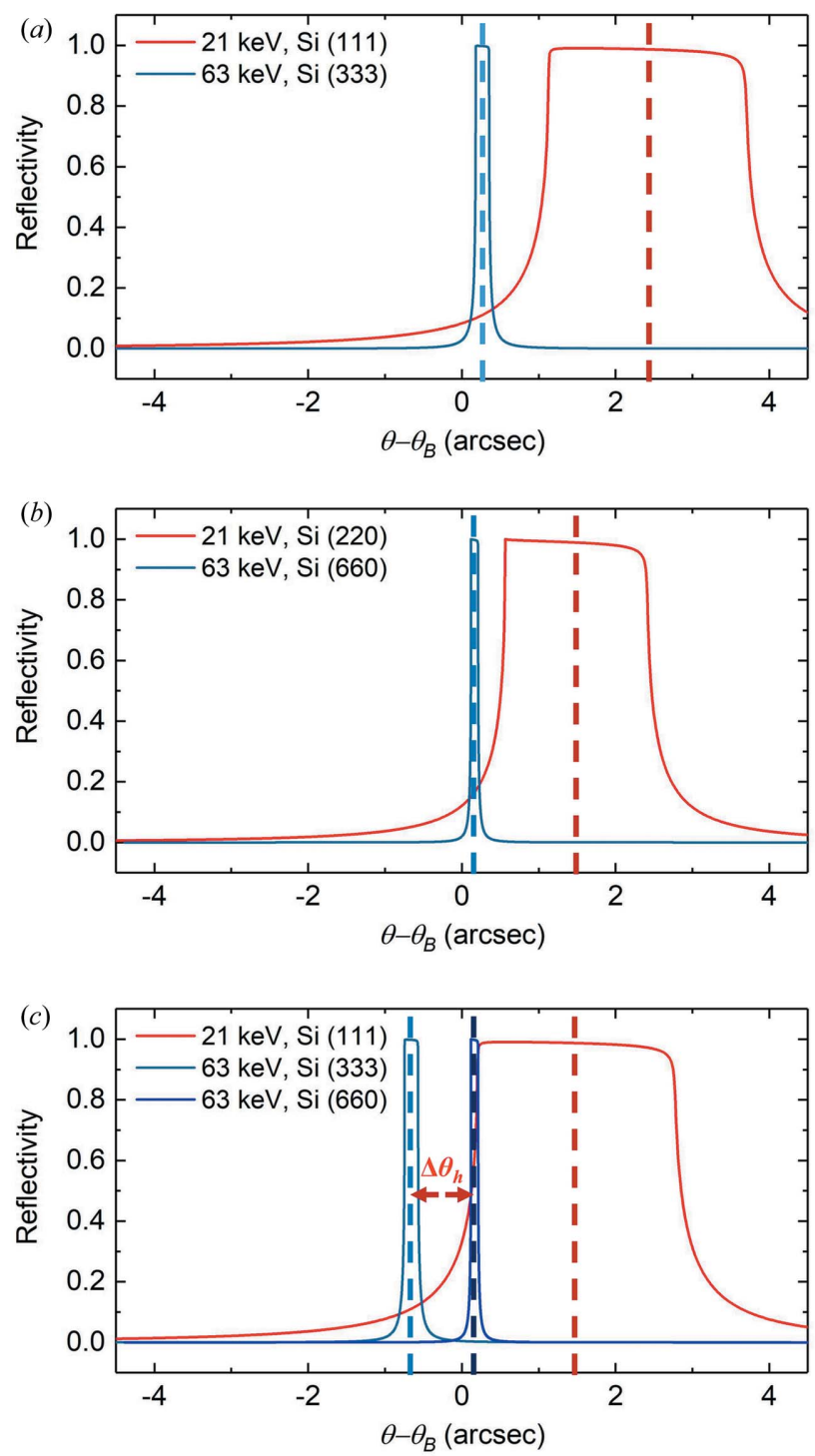

Figure 2

Calculated crystal Darwin curve as a function of angular offset from the Bragg angle for (a) $21 \mathrm{keV}$ X-rays from $\mathrm{Si}(111)$ crystal planes and $63 \mathrm{keV}$ X-rays from $\mathrm{Si}(333)$ crystal planes; (b) $21 \mathrm{keV}$ X-rays from $\mathrm{Si}(220)$ crystal planes and $63 \mathrm{keV}$ X-rays from $\mathrm{Si}(660)$ crystal planes. The red dashed lines in $(a)$ and $(b)$ show the center of the Darwin curves for $21 \mathrm{keV}$ X-ray $\mathrm{s}$ with $\mathrm{Si}(111)$ and $\mathrm{Si}(220)$ crystal planes, respectively. The blue dashed lines in $(a)$ and $(b)$ show the center of the Darwin curves for $63 \mathrm{keV}$ X-ray $\mathrm{s}$ with $\mathrm{Si}(333)$ and $\mathrm{Si}(660)$ crystal planes, respectively. Panel $(c)$ demonstrates that, while maximizing the transmission of the fundamental radiation exiting from the $\mathrm{Si}(111)$ monochromator, the incident angle of the harmonic radiation $(n=3)$ situates at the tail $\left(\Delta \theta_{\mathrm{h}}\right.$ from the center) of the $\mathrm{Si}(660)$ crystal Darwin curve, which leads to the highly effective harmonic-rejection scheme reported in this paper. 
The angular offsets, $\theta_{\mathrm{C}}-\theta_{\mathrm{B}}$, of the $21 \mathrm{keV}$ and $63 \mathrm{keV}$ $\mathrm{X}$-rays from their respective Bragg angles for $\mathrm{Si}(111)$ and $\mathrm{Si}(220)$ optics are different. The dashed lines in Fig. 2 are at the centers of the Darwin curves. The Darwin curves for $21 \mathrm{keV} \mathrm{X}$-rays diffracted by $\mathrm{Si}(111)$ and $63 \mathrm{keV} \mathrm{X}$-rays diffracted by $\mathrm{Si}(333)$ are centered at offsets of 2.4079 arcsec and 0.2671 arcsec, respectively, from the $\theta_{\mathrm{B}}$ values for the $\mathrm{Si}(111) / \mathrm{Si}(333)$ diffraction planes. This leads to a relative angular offset of 2.1408 arcsec. Meanwhile, the Darwin curves for $21 \mathrm{keV}$ X-rays diffracted by $\mathrm{Si}(220)$ and $63 \mathrm{keV} \mathrm{X}$-rays diffracted by $\mathrm{Si}(660)$ are centered at 1.4824 arcsec and $0.1647 \mathrm{arcsec}$, respectively, from the $\theta_{\mathrm{B}}$ values for $\mathrm{Si}(220) /$ $\mathrm{Si}(660)$ diffraction planes, leading to a relative-angular offset of $1.3176 \mathrm{arcsec}$. This allowed a relative offset between $21 \mathrm{keV}$ and $63 \mathrm{keV} \mathrm{X}$-rays arriving at the harmonic-rejection crystal pair that clearly does not match the relative offset allowed and transmitted for these same X-ray energies by the monochromator.

The X-rays generated by an APS undulator A source are collimated with an angular spread. The angular divergence has a Gaussian width on the scale of $15 \mu \mathrm{rad}(3.1 \mathrm{arcsec})$ (Dejus et al., 1994). The flux of the X-ray beam is optimized for fundamental radiation. In our scheme (Fig. 1) the undulator beam is first received by the $\mathrm{Si}(111)$ monochromator. The monochromator is set to monochromatize the fundamental radiation, i.e. the incident angle is close to the Bragg angle $\theta_{\mathrm{B}}$, as dictated by Bragg's law. Other than the harmonic radiation, X-rays of almost all other energies are not transmitted through the monochromator. Darwin curves also serve as angular filters. As Fig. 2(a) clearly shows, the outgoing directions of the fundamental radiation and harmonic radiation are slightly different. In the case of $21 \mathrm{keV}$, this angular difference is 2.1408 arcsec.

After exiting the $\mathrm{Si}(111)$ monochromator, the $\mathrm{X}$-rays, which now consist of fundamental radiation with wavelength $\lambda$ and harmonic radiation with wavelength $\lambda / 3$ (neglecting harmonic radiation resulting from higher-order diffraction), enter the $\mathrm{Si}(220)$ harmonic-rejection crystals. In our scheme, we seek to maximize the intensity of the fundamental radiation after the $\mathrm{X}$-rays exit the $\mathrm{Si}(220)$ crystals. To achieve this, the incident angle of the fundamental radiation entering the $\mathrm{Si}(220)$ optics must be centered on the center of the $\mathrm{Si}(220)$ Darwin curve for the same energy. This is demonstrated by the red dashed lines in Figs. 2(b) and 2(c). The $\mathrm{Si}(111)$ acceptance angle is larger than that of $\mathrm{Si}(220)$, so the $\mathrm{Si}(220)$ Darwin curve is fully populated; this offsets the incident angle of the harmonic radiation $(\lambda / 3)$ away from the center of the corresponding $\mathrm{Si}(660)$ Darwin curve where it could be transmitted through the harmonic-rejection crystal pair. Again, using $21 \mathrm{keV}$ and $63 \mathrm{keV} \mathrm{X}$-rays as an example, the angular difference between the blue dashed lines in Figs. 2(b) and 2(c) is 0.8231 arcsec, approximately 8.5 times the FWHM of the $\mathrm{Si}(660)$ Darwin curve at $63 \mathrm{keV}(0.0973 \mathrm{arcsec})$.

Following this scheme mathematically, the transmission of the harmonic radiation through the harmonic-rejection crystals is defined as

$$
T_{\mathrm{h}}=\frac{\int_{-\infty}^{\infty} I_{\mathrm{h}}(\theta) R_{(333)}^{2}\left(\theta+\Delta \theta_{\mathrm{h}}\right) R_{(660)}^{4}(\theta) \mathrm{d} \theta}{\int_{-\infty}^{\infty} I_{\mathrm{h}}(\theta) R_{(333)}^{2}(\theta) \mathrm{d} \theta},
$$

where $I_{\mathrm{h}}(\theta)$ is the flux of the incident beam at the monochromator and $R_{(333)}(\theta)$ and $R_{(660)}(\theta)$ are the $\operatorname{Si}(333)$ and $\mathrm{Si}(660)$ Darwin curves for harmonic radiation. $\Delta \theta=\theta_{(111)}^{\mathrm{c}}-$ $\theta_{(220)}^{\mathrm{c}}$, where $\theta_{(111)}^{\mathrm{c}}$ and $\theta_{(220)}^{\mathrm{c}}$ are the center of the $\operatorname{Si}(111)$ and $\mathrm{Si}(220)$ Darwin curves of the fundamental radiation, exampled by the red dashed lines in Figs. $2(a)$ and $2(b)$, respectively. Consequently, $\Delta \theta_{\mathrm{h}}=\theta_{(111)}^{\mathrm{c}}-\theta_{(220)}^{\mathrm{c}}-\theta_{(333)}^{\mathrm{c}}+\theta_{(660)}^{\mathrm{c}}$. Here, $\theta_{(333)}^{\mathrm{c}}$ and $\theta_{(660)}^{\mathrm{c}}$ are the centers of the $\operatorname{Si}(333)$ and $\operatorname{Si}(660)$ Darwin curves for fundamental radiation, as shown by the blue dashed lines in Figs. 2(a) and 2(b). Similarly, the transmission of the fundamental radiation from the harmonic-rejection crystals can be calculated by replacing $R_{(333)}(\theta)$ and $R_{(660)}(\theta)$ with $R_{(111)}(\theta)$ and $R_{(220)}(\theta)$ in equation (4), respectively.

One key characteristic of parallel crystal optics is the powerlaw reduction of the diffracted intensity in the tails of the reflectivity curves, resulting from multiple diffractions by parallel perfect-crystal optics (Bonse \& Hart, 1965). Our scheme takes advantage of this characteristic, where the incident angle of the harmonic radiation at the harmonicrejection crystal pair occurs at the tail of the corresponding $\mathrm{Si}(660)$ Darwin curve that represents the acceptance window for diffraction, as illustrated in Figs. 2(b) and 2(c). In our calculation, we further assume that the beam incident at the monochromator is uniform in its angular intensity distribution, i.e. $I_{\mathrm{h}}(\theta)$ is independent of $\theta$. Thus, $I_{\mathrm{h}}(\theta)$ in equation (4) cancels out, and the transmission of the third-harmonic X-rays can be calculated using the theoretical Darwin curves alone.

Fig. 3 shows the calculated transmission of the fundamental and harmonic radiations using the harmonic-rejection crystal pair in a fundamental-radiation energy range from $7 \mathrm{keV}$ to $25 \mathrm{keV}$. The transmission of the fundamental radiation shows a monotonic increase (improved throughput) with increasing $\mathrm{X}$-ray energy. In general, within this energy range, $>50 \%$ of the desired fundamental radiation is allowed through the harmonic-rejection crystal pair. The transmission of the harmonic radiation is on the order of $10^{-6}$, and becomes smaller with increasing X-ray energy. The sharp contrast between the transmissions of the fundamental and harmonic radiations clearly demonstrates that the harmonic radiation is preferentially and effectively suppressed. This is assisted by the fact that the incident flux of the harmonic radiation at the monochromator is already a few orders of magnitude lower than that of the fundamental radiation, owing to the undulator radiation characteristics. This fact, combined with the $10^{-6}$ transmission of the harmonic radiation demonstrated here, leads to an even smaller flux ratio between the harmonic radiation and fundamental radiation after the harmonicrejection crystal pair. This will be discussed further in the experimental section.

It is worth noting that the forbidden $\mathrm{Si}(222)$ reflection from the monochromator does have a very small intensity as a result 


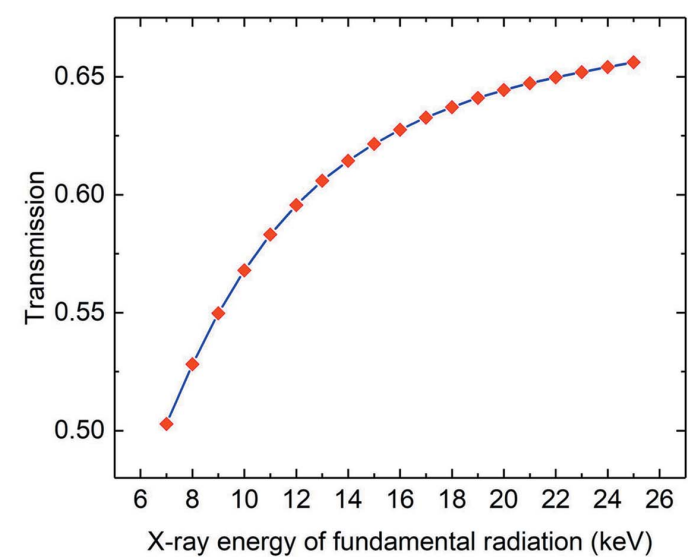

(a)

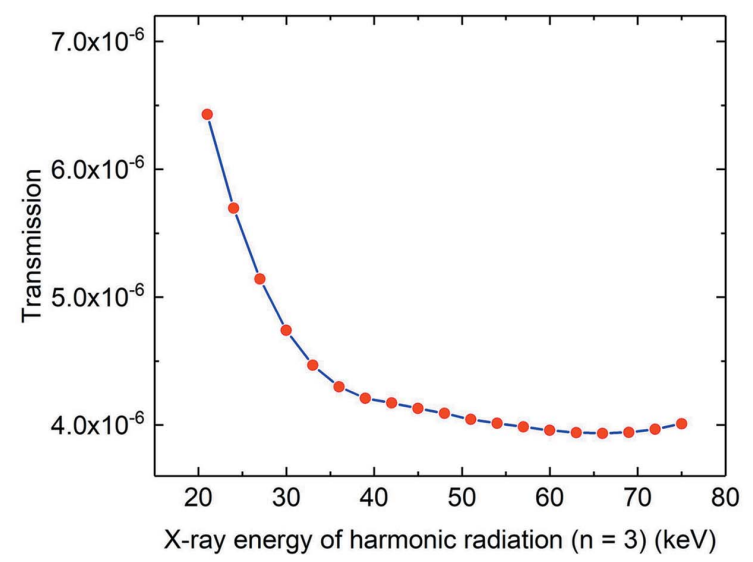

(b)

Figure 3

Calculated energy-dependent transmission of $(a)$ the fundamental radiation and $(b)$ the harmonic radiation $(n=3)$ using the scheme shown in Fig. 1. This transmission is defined as the flux ratio of the outgoing to the incoming incident harmonic radiation for the harmonic-rejection crystal pair.

of the asymmetric distribution of the electronic structure, and this was not explicitly included in the above analysis. However, the small intensity of this forbidden reflection $\left(\sim 10^{-3}\right)$, coupled with the harmonic-rejection scheme described above, results in a transmitted intensity that is significantly $<10^{-6}$.

Although the harmonic rejection is already effective using the scheme shown in Fig. 1, if more harmonic rejection is required, for example, to exploit opportunities for new instrument designs, the scheme in Fig. 1 can be adjusted to achieve this. Instead of having two diffractions by the $\operatorname{Si}(111)$ monochromator crystals, we can use four. Consequently, the power of $R_{(333)}(\theta)$ in equation (4) changes from two to four. The transmission of the fundamental radiation is nearly unchanged as this transmission is largely determined by the relative width of the Darwin curves for $\mathrm{Si}(111)$ and $\mathrm{Si}(220)$ at the energy corresponding to the fundamental radiation. The transmission of the harmonic radiation, however, decreases by another five orders of magnitude to $10^{-11}$, as shown in Fig. 4.

We point out that the key for our scheme to work is the dispersion between the $\mathrm{Si}(111)$ optics in the monochromator and the $\mathrm{Si}(220)$ optics in the harmonic-rejection crystal pair. Should both crystals be of the same type, for example $\mathrm{Si}(111)$,

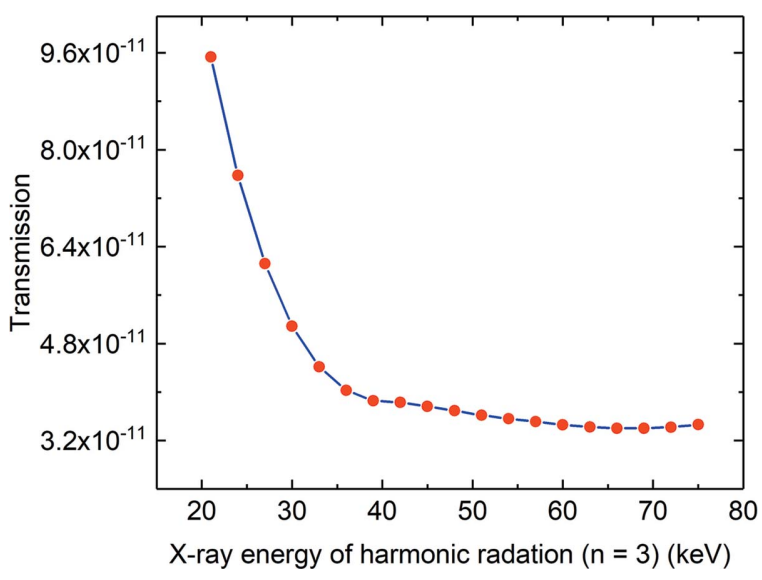

Figure 4

Calculated energy-dependent transmission of the $(n=3)$ harmonic X-rays using a modified scheme with four diffractions by the Si(111) monochromator crystals, as well as four diffractions by the $\operatorname{Si}(220)$ harmonic-rejection crystal pair. The harmonic transmission is defined as the flux ratio of the outgoing to the incoming harmonic radiation incident at the harmonic-rejection crystal pair. Compared with Fig. 3(b), it is apparent that the transmission of the $(n=3)$ harmonic X-rays is reduced by another five orders of magnitude by introducing two additional reflections in the $\mathrm{Si}(111)$ monochromator.

$\Delta \theta$ in equation (4) becomes 0 , and both the fundamental and harmonic radiation that exit from the monochromator will be accepted by the angular filters imposed by the harmonicrejection crystal pair. Thus, effective harmonic rejection will not be achieved under these conditions.

\section{Experimental verification: harmonic transmission}

We measured the transmission of the fundamental and harmonic radiations for our scheme using the ultra-smallangle X-ray scattering (USAXS) instrument at APS sector 9ID (Ilavsky et al., 2009, 2018). This beamline makes use of an APS undulator A source and a Si(111) double-crystal monochromator. Importantly, the USAXS instrument is equipped with nondispersive Bonse-Hart-type $\mathrm{Si}(220)$ crystal optics to collimate the incident X-rays. $\mathrm{Si}(220)$ crystal has the advantage of a narrower rocking curve when compared with $\mathrm{Si}(111)$, which results in a better collimation (Diat et al., 1997). Each $\mathrm{Si}(220)$ crystal, instead of being part of a channel-cut crystal, is independently precision-mounted on a three-point kinematic mount attached to a rotation stage and controlled by means of an over-constrained weak-link mechanism with picomotor and piezoelectric transducers serving as actuators (Shu et al., 2001).

We probed the harmonic transmission of the harmonicrejection crystals using an energy-sensitive Vortex X-ray detector (Hitachi, Chatsworth, CA, USA). ${ }^{\mathbf{1}}$ In our experiment, we chose the X-ray energies of $21 \mathrm{keV}$ and $63 \mathrm{keV}$ for the

\footnotetext{
${ }^{\mathbf{1}}$ Certain commercial equipment, instruments or materials (or suppliers or software) are identified in this paper to foster understanding. Such identification does not imply recommendation or endorsement by the National Institute of Standards and Technology, nor does it imply that the materials or equipment identified are necessarily the best available for the purpose.
} 
Table 2

Experimental parameters for measuring harmonic transmission of the harmonic-rejection crystal pair using a Vortex energy-sensitive detector. Uncertainties in the Vortex detector counts follow standard Poisson statistics behavior.

\begin{tabular}{|c|c|c|c|}
\hline & $\begin{array}{l}\text { Beam size } \\
(\mathrm{mm} \times \mathrm{mm})\end{array}$ & Attenuation & $\begin{array}{l}\text { Vortex detector counts of } \\
63 \mathrm{keV} \text { X-rays ( } 5 \mathrm{~s} \text { counting time) }\end{array}$ \\
\hline Without harmonic-rejection crystals & $0.05 \times 0.10$ & $3.75 \mathrm{~mm} \mathrm{Ti}+3.75 \mathrm{~mm} \mathrm{Al}+2$ layers of lead tape & $1.25 \times 10^{4}$ \\
\hline With harmonic-rejection crystals & $1.00 \times 1.00$ & $3.75 \mathrm{~mm} \mathrm{Ti}+3.75 \mathrm{~mm} \mathrm{Al}+2$ layers of lead tape & 6 \\
\hline
\end{tabular}

fundamental and harmonic radiation, respectively. We focused on the intensity of the harmonic radiation, and measured the $63 \mathrm{keV}$ intensity with and without the harmonic-rejection crystals in the beam. These results are tabulated in Table 2.

With the harmonic-rejection crystals out of the beam, the Vortex detector measures the intensity of incoming thirdharmonic X-rays from the monochromator as illustrated by the red beam in Fig. 1. To avoid saturating the detector, we set the beam size at $0.05 \mathrm{~mm} \times 0.10 \mathrm{~mm}$, and used all of the Ti and Al filters available for everyday operation at the beamline as well as two layers of lead tape to attenuate the X-ray intensity. A $5 \mathrm{~s}$ exposure produced a detector readout of $1.25 \times 10^{4}$.

With the harmonic-rejection crystals in the beam, the Vortex detector measures the incoming intensity of the harmonic radiation as illustrated by the green beam in Fig. 1. For this measurement, we used a beam size of $1.00 \mathrm{~mm} \times$ $1.00 \mathrm{~mm}$ and the same amount of beam attenuation. With this beam, a $5 \mathrm{~s}$ exposure produced a detector readout of 6 . The Vortex detector is a photon-counting device, and its background level is effectively zero. Assuming uniform illumination, a normalization of detector readouts using the beam size yields that the harmonic-rejection crystals have a transmission of $6 /(1.00 \mathrm{~mm} \times 1.00 \mathrm{~mm}) /\left[1.25 \times 10^{4} /(0.05 \mathrm{~mm} \times 0.10 \mathrm{~mm})\right]$ $=2.4 \times 10^{-6}$. Allowing for measurement uncertainties, including those for the Vortex detector counts which exhibit Poisson statistics, this is in reasonable agreement with the theoretical transmission of $3.9 \times 10^{-6}$. It should also be considered that any discrepancy between the measured and predicted values can also arise from the assumptions made concerning the spatial and angular uniformity of the incident $\mathrm{X}$-ray beam, any slight imperfections in the crystal optics (the theoretical calculations assume perfect crystals), as well as imperfect alignment between the two crystals along their tilt directions (Allen et al., 2017; Ilavsky et al., 2009).

Although the experiment using an energy-dispersive detector clearly demonstrates that our scheme is highly effective in removing the harmonic radiation, it does not provide information regarding the intensity ratio of fundamental and harmonic radiations in the incoming beam (red beam in Fig. 1). We performed a second experiment using a photodiode detector and two sets of X-ray filters. By solving a simple set of linear equations, we estimated that in the incoming beam the flux ratio between $63 \mathrm{keV}$ and $21 \mathrm{keV}$ $\mathrm{X}$-rays is $7.3 \times 10^{-5}$ (details about this calculation can be found in the supporting information). Considering the theoretical transmission for the $21 \mathrm{keV}$ X-rays after four reflections from $\mathrm{Si}(220)$ crystals is approximately 0.65 , we arrived at an estimated flux ratio between $63 \mathrm{keV}$ and $21 \mathrm{keV}$ X-rays in the outgoing beam (green beam in Fig. 1) of the harmonicrejection crystal pair to be $2.7 \times 10^{-10}$ using our setup. Importantly, we emphasize that using an X-ray camera we routinely observe positional offset between the fundamental and harmonic radiations emerging from the monochromator. The vertical positional offset observed (typically $\sim 0.5 \mathrm{~mm}$ at the harmonic-rejection crystals) is both a manifestation of the angular dispersion between the fundamental and harmonic radiations, indicated in Fig. 2(a), and is within expectations, given the distance between the monochromator and the harmonic-rejection crystals. This observation provides yet another piece of evidence supporting our proposed harmonicrejection mechanism.

\section{Coherence preservation}

For the next-generation synchrotron X-ray sources, one significant breakthrough will be the dramatic increase in beam coherence. As discussed in the Introduction, wavefront distortion introduced by optical elements in the beam path adversely affects coherent X-ray applications such as coherent diffraction imaging and X-ray photon correlation spectroscopy (XPCS). These techniques place a particularly high requirement on the finish of conventional harmonic-rejection mirrors. At the same time, double-crystal optics are known not only to remove unwanted parasitic scattering but also to preserve the coherent wave propagation (Xiao et al., 2006). Following this concept, we performed XPCS experiments to qualitatively estimate the effect that harmonic-rejection crystals have on the beam coherence.

The setup for these USAXS-based XPCS experiments has been described previously (Zhang et al., 2011). The sample used for this study was a monodispersed $\mathrm{SiO}_{2}$ colloidal suspension, prepared following an established protocol (Zhang et al., 2017). The diameter of the $\mathrm{SiO}_{2}$ particles was approximately $500 \mathrm{~nm}$. The equilibrium dynamics of this colloidal suspension can be characterized using the normalized intensity autocorrelation function $g_{2}(t)$, defined as following its ensemble average:

$$
g_{2}(q, t)=\frac{\left\langle I\left(q, t+t^{\prime}\right) I\left(q, t^{\prime}\right)\right\rangle_{\mathrm{E}}}{\left\langle I\left(q, t^{\prime}\right)\right\rangle_{\mathrm{E}}{ }^{2}} .
$$

Here, $I(q, t)$ is the time $(t)$ dependent detector intensity normalized by ion-chamber readout acquired at a fixed $q$. When the sample contains a large number of independent scatterers undergoing equilibrium thermal motion, following the central limit theorem, the temporal fluctuations of the 
coherent scattering intensity obey Gaussian statistics and $g_{2}(q, t)$ can be related to the intermediate scattering function of the sample following the Siegert relation (Goodman, 1985):

$$
g_{2}(q, t)=1+\beta|f(q, t)|^{2}
$$

where $f(q, t)=S(q, t) / S(q)$ is the normalized intermediate scattering function with $S(q)$ and $S(q, t)$ being the static and time-dependent dynamic structure factors at time $t$, respectively. An important conclusion can be drawn from equation (6), which is that as $t^{\prime} \rightarrow 0, g_{2}(q, t) \rightarrow 1+\beta$. The parameter $\beta$ is known as the optical contrast and accounts for the smearing that is introduced by scattering from a volume larger than one coherence volume. In other words, $\beta$ provides a quantitative measure that allows the beam coherence to be estimated and compared.

To interrogate the effectiveness that the harmonic-rejection crystals have on preserving the beam coherence, we performed our experiments at $10.5 \mathrm{keV}$, a previously identified optimal energy for XPCS work at the USAXS beamline (Zhang et al., 2011). Based on the beam characteristics, we calculated that at $10.5 \mathrm{keV}$ the horizontal and vertical coherence lengths at the beamline are $3.5 \mu \mathrm{m}$ and $35 \mu \mathrm{m}$, respectively.

To test the effect of harmonic-rejection crystal optics on the measured coherence, we used a set of high-resolution JJ slits (JJ slit, Rosskilde, Denmark) as the secondary coherence source, adjusted the vertical and horizontal slit sizes systematically, and characterized the corresponding optical contrasts. The results are shown in Table 3, from which we conclude that, with the harmonic-rejection optics in the beam at a fixed vertical slit size, increasing horizontal slit size leads to a significant decrease in the optical contrast, owing to the very small value of the horizontal coherence length. In contrast, at a fixed horizontal slit size, the optical contrast is not sensitive to the changes in the vertical slit size until its value greatly exceeds the vertical coherence length. In other words, the beam coherence follows the expected pattern, indicating that the X-ray coherence is preserved, at least qualitatively.

\section{Conclusions}

In this paper, we have described a simple harmonic-rejection scheme that exploits a mismatch in the crystal Bragg diffractions between those in the monochromator crystals and those in the Bonse-Hart-type harmonic-rejection crystal pair. Our scheme makes use of two reflections by the $\mathrm{Si}(111)$ monochromator crystals and four reflections by the $\mathrm{Si}(220)$ harmonic-rejection crystals. Dynamical diffraction theory dictates that, for a given crystal, the Darwin curves of fundamental and harmonic radiations have different angular offsets from the Bragg angle $\theta_{\mathrm{B}}$. Furthermore, their relative offset is different for different crystal reflections. Meanwhile, multiple reflections by single-crystal optics substantially reduce the reflectivity in the tails of the reflectivity curves. By maximizing the transmission of the fundamental radiation, our calculation shows that the allowed transmission of the harmonic radiation
Table 3

Optical contrast as a function of the horizontal and vertical dimensions of the coherence defining slits.

\begin{tabular}{lll}
\hline $\begin{array}{l}\text { Vertical slit } \\
\text { size }(\mu \mathrm{m})\end{array}$ & $\begin{array}{l}\text { Horizontal slit } \\
\text { size }(\mu \mathrm{m})\end{array}$ & Optical contrast \\
\hline 15 & 15 & $0.044(2)$ \\
15 & 30 & $0.031(2)$ \\
15 & 50 & $0.018(1)$ \\
15 & 100 & $0.0091(5)$ \\
30 & 15 & $0.036(2)$ \\
50 & 15 & $0.035(2)$ \\
100 & 15 & $0.012(1)$ \\
30 & 30 & $0.023(1)$ \\
50 & 50 & $0.0094(5)$ \\
100 & 100 & $0.0067(3)$ \\
\hline
\end{tabular}

$(n=3)$ by the harmonic-rejection crystals is on the order of $10^{-6}$. Considering an experimentally measured $10^{-5}$ flux ratio between harmonic radiation and fundamental radiation incident on the harmonic-rejection crystals, our scheme shows that the flux ratio of the harmonic radiation to the fundamental radiation exiting the harmonic-rejection crystals is on the order of $10^{-10}$. This level of harmonic-rejection is expected to be adequate for the requirements of most, if not all, synchrotron experiments. Minor changes to the monochromator design, making use of four instead of two reflections, would provide an additional five orders of magnitude harmonic rejection.

Using the two-reflection monochromator setup, we performed a qualitative evaluation of the spatial coherence of the X-ray beam while adjusting the beam size systematically. We found that the beam coherence follows the expected behavior, strongly indicating that the coherence of the beam is preserved. This setup is also highly stable. In practice, the X-ray beam after the harmonic-rejection crystals can remain stable for hours without the need of retuning.

We have successfully used this scheme at two synchrotron beamlines at the Advanced Photon Source (sector 9-ID and previously sector 15-ID) to perform small-angle X-ray scattering (SAXS), USAXS, XPCS and imaging experiments. Although both beamlines are equipped with harmonic-rejection mirrors, the mirrors are no longer part of the beamline optics for USAXS operations. These different techniques have very different requirements. Both USAXS and SAXS seek a high dynamic range (over ten decades in scattering intensity in the case of USAXS). Because of this, harmonic contamination is a serious concern (Long et al., 1991). Using this setup, we performed SAXS using a Pilatus $100 \mathrm{~K}$ detector and performed USAXS using a high-dynamic-range photodiode detector with an additional pair of Bonse-Hart optics as analyzing crystals. In both types of scattering experiments, no contamination has been detected. We also found that this setup preserves the coherence of the beam for XPCS experiments and removes undesired imaging footprints and distortions caused by the mirror stripes, thus serving to improve data quality and integrity.

When used in place of a harmonic-rejection mirror, this scheme bypasses the increasingly stringent requirements 
placed on the surface of X-ray mirrors to ensure coherence preservation for applications associated with highly coherent $\mathrm{X}$-rays produced by diffraction-limited storage rings and $\mathrm{X}$-ray free-electron lasers. With an $\mathrm{Si}(111)$ monochromator already a required component of many beamlines, the cost for implementing this scheme resides only in the harmonicrejection crystal pair and its assembly. With the added benefits of coherence preservation and small footprint, we envisage that this highly effective scheme could serve the harmonicrejection needs of the broad synchrotron community in the era of diffraction-limited storage rings and beyond.

\section{Acknowledgements}

We thank Elina Kasman for preparing the crystals used in this study.

\section{Funding information}

Use of the Advanced Photon Source, an Office of Science User Facility operated for the US Department of Energy (DOE) by Argonne National Laboratory, was supported by the US DOE under Contract No. DE-AC0206CH11357.ChemMatCARS. Sector 15 is principally supported by the National Science Foundation/Department of Energy under grant No. NSF/CHE-0822838.

\section{References}

Allen, A. J., Zhang, F., Kline, R. J., Guthrie, W. F. \& Ilavsky, J. (2017). J. Appl. Cryst. 50, 462-474.

Als-Nielsen, J. \& McMorrow, D. (2011). Elements of Modern X-ray Physics. Chichester: Wiley.

Bilderback, D. \& Hubbard, S. (1982). Nucl. Instrum. Methods Phys. Res. 195, 91-95.

Bonse, U. \& Hart, M. (1965). Appl. Phys. Lett. 7, 238-240.

Bonse, U., Materlik, G. \& Schröder, W. (1976). J. Appl. Cryst. 9, $223-$ 230.

Cromer, D. T. \& Liberman, D. A. (1981). Acta Cryst. A37, 267-268.

Dejus, R. J., Lai, B., Moog, E. R. \& Gluskin, E. (1994). Undulator A Characteristics and Specifications: Enhanced Capabilities. Argonne National Laboratory, Argonne, IL, USA.
Diat, O., Bösecke, P., Lambard, L. \& de Moor, P. P. E. A. (1997). J. Appl. Cryst. 30, 862-866.

Gauthier, C., Solé, V. A., Signorato, R., Goulon, J. \& Moguiline, E. (1999). J. Synchrotron Rad. 6, 164-166.

Goodman, J. W. (1985). Statistical Optics. New York: Wiley.

Hastings, J., Kincaid, B. \& Eisenberger, P. (1978). Nucl. Instrum. Methods, 152, 167-171.

Hou, Z. (2005). Rev. Sci. Instrum. 76, 013305.

Ilavsky, J. \& Jemian, P. R. (2009). J. Appl. Cryst. 42, 347-353.

Ilavsky, J., Jemian, P. R., Allen, A. J., Zhang, F., Levine, L. E. \& Long, G. G. (2009). J. Appl. Cryst. 42, 469-479.

Ilavsky, J., Zhang, F., Andrews, R. N., Kuzmenko, I., Jemian, P. R., Levine, L. E. \& Allen, A. J. (2018). J. Appl. Cryst. 51, 867882.

Karanfil, C., Chapman, D., Segre, C. U. \& Bunker, G. (2004). J. Synchrotron Rad. 11, 393-398.

Latimer, M. J., Rompel, A., Underwood, J. H., Yachandra, V. K. \& Klein, M. P. (1995). Rev. Sci. Instrum. 66, 1843-1845.

Lingham, M., Ziegler, E., Luken, E., Loeffen, P. W., Muellender, S. \& Goulon, J. (1996). Proc. SPIE, 2805, 158-168.

Long, G. G., Jemian, P. R., Weertman, J. R., Black, D. R., Burdette, H. E. \& Spal, R. (1991). J. Appl. Cryst. 24, 30-37.

Mills, D. \& Padmore, H. (2013). X-ray Optics for BES Light Source Facilities. US DOE Office of Science, USA.

Pardini, T., Cocco, D. \& Hau-Riege, S. P. (2015). Opt. Express, 23, 31889-31895.

Paterson, D., de Jonge, M., Howard, D., Lewis, W., McKinlay, J., Starritt, A., Kusel, M., Ryan, C., Kirkham, R., Moorhead, G., Siddons, D. P., McNulty, I., Eyberger, C. \& Lai, B. (2011). AIP Conf. Proc. 1365, 219-222.

Polikarpov, M., Snigireva, I. \& Snigirev, A. (2014a). J. Synchrotron Rad. 21, 484-487.

Polikarpov, M., Snigireva, I. \& Snigirev, A. (2014b). Proc. SPIE, 9207, 920711.

Shu, D., Toellner, T. S. \& Alp, E. E. (2001). Nucl. Instrum. Methods Phys. Res. A, 467-468, 771-774.

Tanaka, T. \& Kitamura, H. (2002). J. Synchrotron Rad. 9, 266269.

Xiao, X., de Jonge, M. D., Zhong, Y., Chu, Y. S. \& Shen, Q. (2006). Opt. Lett. 31, 3194-3196.

Zhang, F., Allen, A. J., Levine, L. E., Ilavsky, J., Long, G. G. \& Sandy, A. R. (2011). J. Appl. Cryst. 44, 200-212.

Zhang, F., Allen, A. J., Levine, L. E., Tsai, D.-H. \& Ilavsky, J. (2017). Langmuir, 33, 2817-2828.

Zhang, F., Long, G. G., Levine, L. E., Ilavsky, J. \& Jemian, P. R. (2008). J. Appl. Cryst. 41, 416-427. 